\title{
Assessing Fan Flutter Stability in Presence of Inlet Distortion Using One-way and Two-way Coupled Methods
}

\author{
Gregory P. Herrick* \\ NASA Glenn Research Center, Cleveland, Ohio, 44135, USA
}

\begin{abstract}
Concerns regarding noise, propulsive efficiency, and fuel burn are inspiring aircraft designs wherein the propulsive turbomachines are partially (or fully) embedded within the airframe; such designs present serious concerns with regard to aerodynamic and aeromechanic performance of the compression system in response to inlet distortion. Previously, a preliminary design of a forward-swept high-speed fan exhibited flutter concerns in cleaninlet flows, and the present author then studied this fan further in the presence of off-design distorted in-flows. Continuing this research, a three-dimensional, unsteady, Navier-Stokes computational fluid dynamics code is again applied to analyze and corroborate fan performance with clean inlet flow and now with a simplified, sinusoidal distortion of total pressure at the aerodynamic interface plane. This code, already validated in its application to assess aerodynamic damping of vibrating blades at various flow conditions using a one-way coupled energy-exchange approach, is modified to include a two-way coupled time-marching aeroelastic simulation capability. The two coupling methods are compared in their evaluation of flutter stability in the presence of distorted in-flows.
\end{abstract}

\section{Nomenclature}

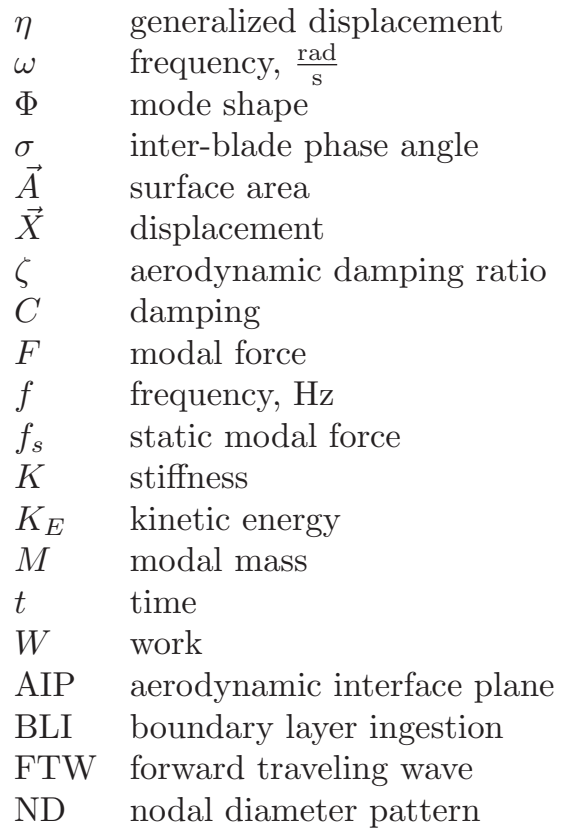

\footnotetext{
${ }^{*}$ Research Aerospace Engineer, LMS/Multiscale \& Multiphysics Modeling Branch, 21000 Brookpark Road, Mail Stop 49-8, AIAA Member
} 


\section{Introduction}

CINCE the advent of turbojet propulsion, "clean" intake flow for the fan (or propulsor) of the gas turbine Nengine has been the desired, elusive, goal of airframe, inlet, and propulsion system designers alike in the quest for optimal performance of the propulsion system. As we look to the future, design goals now focus on reduced emissions, reduced fuel consumption, better propulsive efficiency (of the entire aircraft system), and reduced ambient noise. Among the prominent design concepts under development for application to future aircraft is the "Blended Wing Body" (alternatively known as "hybrid wing/body"). Most of these prospective designs feature propulsion systems wherein the propulsive fan, whether coupled directly to a Brayton cycle turbine engine or part of a turbo-electric distributed propulsion system, is partially or fully embedded within the body.

Boundary layer ingestion (BLI) propulsion has the potential for significant reduction in aircraft fuel burn ${ }^{1}$ previous system studies ${ }^{2-6}$ have shown that $5-10 \%$ reduction in fuel burn is possible. Recent work ${ }^{7}$ has confirmed this potential benefit through a system study focused on the propulsion system and its integration into the BWB aircraft. The system study indicated that low-loss inlets and high-performance, distortiontolerant turbomachinery are key technologies required to achieve a 3-5\% BLI fuel burn benefit for future aircraft relative to a baseline high-performance, pylon-mounted, propulsion system. A robust aeromechanical design with respect to dynamic stresses and flutter stability in the presence of distortion is critical to the successful implementation and application of BLI propulsion systems.

In previous work from Herrick, ${ }^{8}$ a parallel computational fluid dynamics (CFD) code was modified and customized to begin studying the issues relevant to aeromechanical response of turbomachinery to distorted inlet flows. In that effort, the aeroelastic analysis employed in the CFD code was "one-way" coupled: The blades' motion was prescribed and enforced, perturbing the flow field, while the resultant changes in blade surface pressures were not fed back to alter the blades' motions. In this effort, a "two-way" coupled method of aeroelastic analysis is implemented into the code and applied in the analysis of the fan subject to distorted inlet flows: The motion of the blades perturbs the flow field, and the flow field's unsteady pressures in turn influence the motion of the blades. Many two-way coupled methods for turbomachinery aeroelasticity have been presented in the literature; $;^{9-11}$ Bréard $^{12}$ et al. applied their integrated model to the study of forced response due to inlet distortion.

\section{Background}

The CFD code modified and applied in this study is TURBO, a physics-based simulation tool for multistage turbomachinery. The solver computes the fluid conservation laws without ad hoc modeling of any flow phenomena other than models required for turbulence. This code solves the unsteady Reynolds-averaged Navier-Stokes equations and a decoupled k- $\epsilon$ turbulence model developed by Zhu and Shih. ${ }^{13}$ The code is implemented in a portable, scalable form for distributed-memory parallel computers using MPI message passing. The parallel implementation employs domain decomposition and supports general multi-block grids with arbitrary grid-block connectivity. The solution algorithm is a Newton iterative implicit time-accurate scheme with characteristics-based finite-volume spatial discretization. The Newton subiterations are solved using a concurrent block-Jacobi symmetric Gauss-Seidel (BJ-SGS) relaxation scheme. Because all of the fundamental fluid mechanics are computed, the code is capable of capturing the nonlinear characteristics of the flow fields of interest. With the actual modeling of grid movement of blade rows in relative motion, this code is capable of computing the unsteady interactions between blade rows. Details of the flow solver are given by Chen and Whitfield. ${ }^{14}$ The approach to parallelization for large-scale, complex problems is discussed by Chen and Briley. ${ }^{15}$ TURBO has been previously modified for application to study flutter and forced vibration. ${ }^{16-18}$ TURBO has been further validated in several previous research efforts regarding aeromechanics. ${ }^{19-26}$

The fan of interest is comprised of twenty-two blades. The fan was designed for purposes of noise reduction in a typical podded-engine mounting. Physically, the average radial tip gap as gridded for this study is about $0.28 \%$ of blade radial span. The fan was never intended to be part of an embedded propulsion system with distorted inlet flow. Previous aeromechanics analyses ${ }^{25}$ of this fan exhibited good corroboration between computational and experimental performance in clean-inlet flow conditions.

In his initial study of this fan's aeroelastic behavior in distorted inlet flows, Herrick ${ }^{8}$ modified the code's total-condition preserved inlet boundary condition to allow for circumferential nonuniformity at the inlet 
plane. Application of a throttle exit boundary condition, allowing the exit flow to attain equilibrium without artificial constraint, showed that inlet distortions do not fully attenuate upon passage through a single rotor. In the previous work, examination of aeroelastic behavior - using a prescribed motion, one-way coupled approach - in clean inlet conditions generally yielded clear, consistent solutions. The distorted inlet aeroelastic simulations (using the same one-way coupled approach, but with an inlet total pressure distribution approximating that of Inlet $\mathrm{A}^{27}$ ) yielded dramatic variation in aerodynamic damping computations about the annulus, with some annular sectors exhibiting great aeroelastic stability and other annular sectors indicating significant aeroelastic instability. These intriguing observations prompt further investigation into the methods used to assess aeroelastic behavior in a distorted flow environment.

This study shall further investigate several issues relevant to the computational assessment of flutter stability for turbomachinery rotors in distorted flow fields: "one-way coupled" versus "two-way coupled" motion of the blades in the unsteady flow field, post-processing/interpretation of computed output (modal force, modal displacement), and initial conditions. The fan is gridded with a single H-block topology for the fan passage, with 214 grid points streamwise, 76 grid points radially, and 58 grid points pitchwise; the blade itself has 81 grid points streamwise and 70 points in the spanwise direction. No tip grid is present: Instead, a lossless multi-point constraint is applied between the suction and pressure sides in the region between the blade's radial extent and the casing. Computationally, the fan is preceded by inlet duct of length-ratio 1.387 with respect to blade-tip axial chord and followed by exit duct of length-ratio 2.365 with respect to blade-tip axial chord. Again, the total-condition preserved boundary condition, capable of enforcing circumferentiallyvarying flow conditions, is applied at the inlet/AIP. The throttle boundary condition is again applied at the exit plane.

To facilitate the goals of this research effort, some fundamental assumptions and simplifications are applied in the study regarding operating speed, inlet distortion pattern, blade mode shape, and blade natural frequency. The fan is operated at $10800 \mathrm{rpm}$, a part-speed condition. From this part-speed condition the fan's aeroelastic behavior is assessed near op-line and near stall. The mode shape and frequency of interest are artificial; its frequency is dictated to be $220 \mathrm{~Hz}$ such that a blade completes one oscillation cycle in the course of traversing precisely 18 blade pitches at $10800 \mathrm{rpm}$. The CFD mesh of the blade, with deflected geometry, is shown in Fig. 1. The artificial mode shape and frequency, in concert with the part-speed run condition, are chosen with intent to encounter stable and unstable flutter conditions.

The total pressure distortion at the inlet plane is a simple, single-frequency, once-per-revoluton spatial distribution. The simulations are performed rotor-alone, and only one mode is studied, thus the only fundamental frequencies at play are the blades' vibrational frequency and the rotor's rotational frequency. The single-frequency, once-per-revolution spatial distortion of total pressure is created by applying a $4 \%$ (mean-to-peak) sinusoidal variation upon the (circumferentially-constant, radially-varying) clean flow total pressure profile in a single wave about the inlet annulus. No circumferential nonuniformity is applied to total temperature or flow angles. Simulations reveal that the sinusoidal distortion yields a $0.12 \%$ higher (energyaveraged) integrated total pressure at the inlet plane than the clean, axisymmetric pressure distribution. The fan characteristic at part-speed is shown in Fig. 2. The total pressure distributions for clean and distorted in-flow are shown in Fig. 3. CFD simulations are executed with 15 time steps per blade pitch; for clean inlet simulations, 6 Newton subiterations are applied at each time step, while 9 Newton subiterations per time step are used for the sinusoidally-distorted inlet runs.

\section{One-way Coupled Method for Aeroelastic Analysis}

\section{A. Description and Implementation}

Flutter stability of the fan is first evaluated using the TURBO aeroelastic analysis code's one-way coupled method. The energy method, ${ }^{28}$ or work-per-cycle approach, is used to calculate aerodynamic damping (and hence flutter stability) as described in Ref. 26; Vasanthakumar ${ }^{29}$ used a similar approach in flutter analysis of a transonic fan. For a clean inflow prescribed at the inlet boundary, the flow field through the blade row is first calculated with no prescribed blade oscillation. Starting with this converged steady flow field, blade vibrations are prescribed in a selected mode, frequency $(f)$, and nodal diameter pattern (ND) or inter-blade phase angle $(\sigma)$. Note that the number of different possible nodal diameter patterns is equal to the number of blades on the rotor of interest. After the transients in the flow field decay and a periodic flow field is 


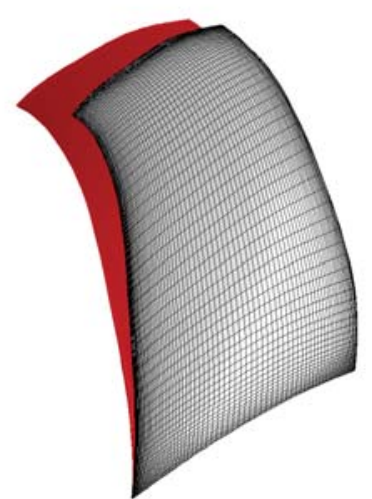

Figure 1: Mesh and deflected shape applied to blade in this research.

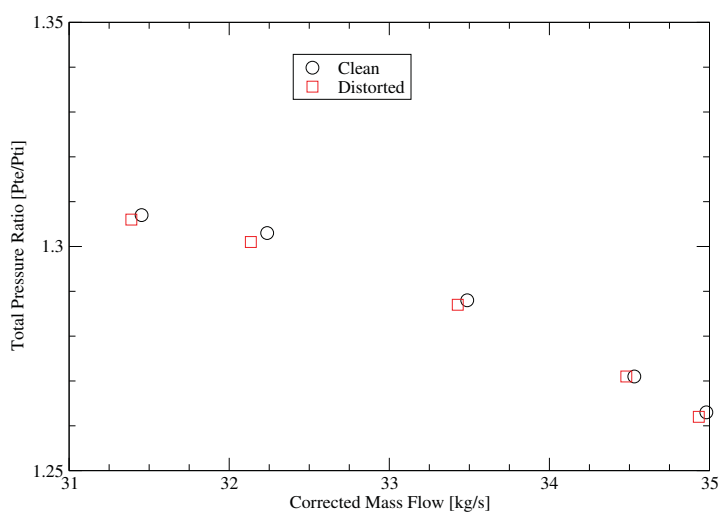

Figure 2: Speedlines: Clean and distorted inlets, partspeed.

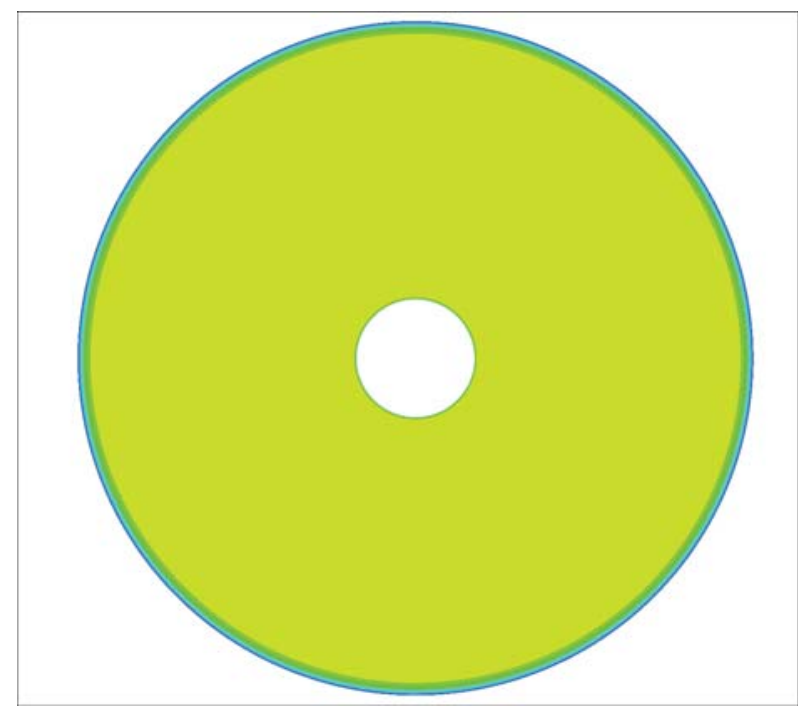

(a) Clean Inlet

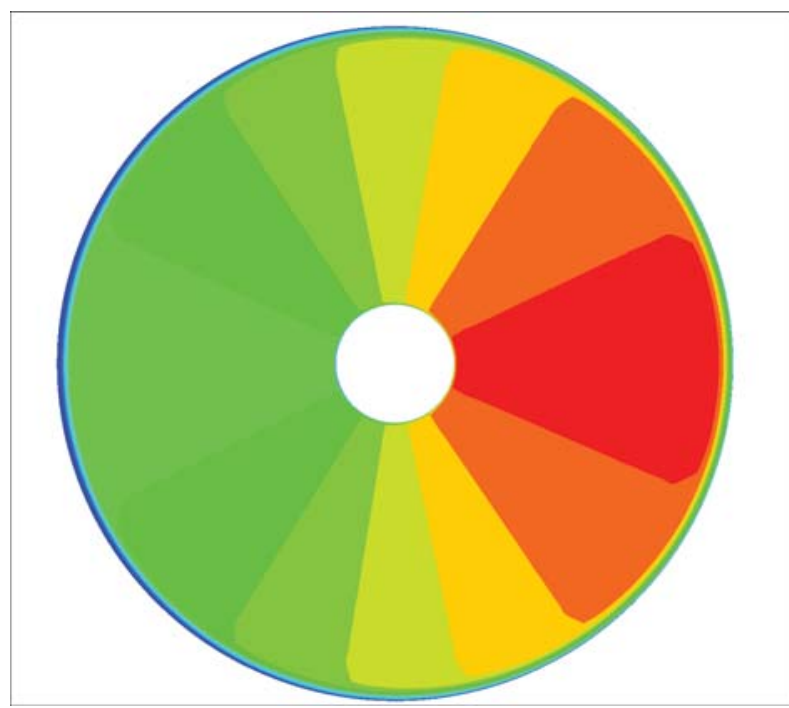

(b) Sinusoidal Inlet Distortion

Figure 3: Inlet total pressure distributions for the clean and distorted inlets of this study. Common colorbar,

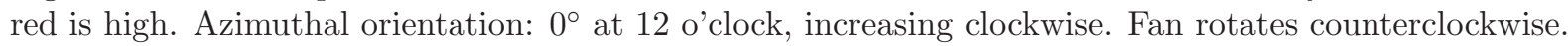

obtained, the work done on the vibrating blade is calculated as follows:

$$
W=-\oint \int_{\text {surface }} p d \vec{A} \cdot \frac{\partial \vec{X}}{\partial t} d t
$$

where $p$ is the aerodynamic pressure, $\vec{A}$ is the blade surface area vector, $\vec{X}$ is the displacement vector on the blade surface, and $t$ denotes time. The aerodynamic damping ratio $(\zeta)$ can be approximately related to the work-per-cycle $(W)$ and the average kinetic energy $\left(K_{E}\right)$ of the blade over one cycle of vibration through the following expression:

$$
\zeta \approx-\frac{W}{8 K_{E}}
$$

The schematic for this method is shown in Fig. 4. If aerodynamic damping is negative (i.e., aerodynamic work is positive), flutter may occur. Note that structural damping (material and mechanical) has not been 
considered.

\section{B. Application to Distorted In-Flows}

In previous research, Herrick ${ }^{8}$ and Bakhle $^{26}$ et al. have utilized the one-way coupled method to assess stability of fans in the presence of distorted inflows. In each of these endeavors, the temporallyand spatially-averaged damping of all blades - a single value attributed to an idealized "average" blade - was used to gauge the flutter stability of the entire rotor in the presence of the circumferentially-varying flow field.

In the presence of a clean-inlet flow field, this single-value average represents very well the consistent response of each and every blade as the blade traverses the flow field. In Fig. 5(a) the convergence time history of the aerodynamic work on all blades is plotted along with the single-value average; in Fig. 5(b), the annulus is "unrolled", and the aerodynamic work distribution for each of the twenty-two blades is plotted versus each blade's re-

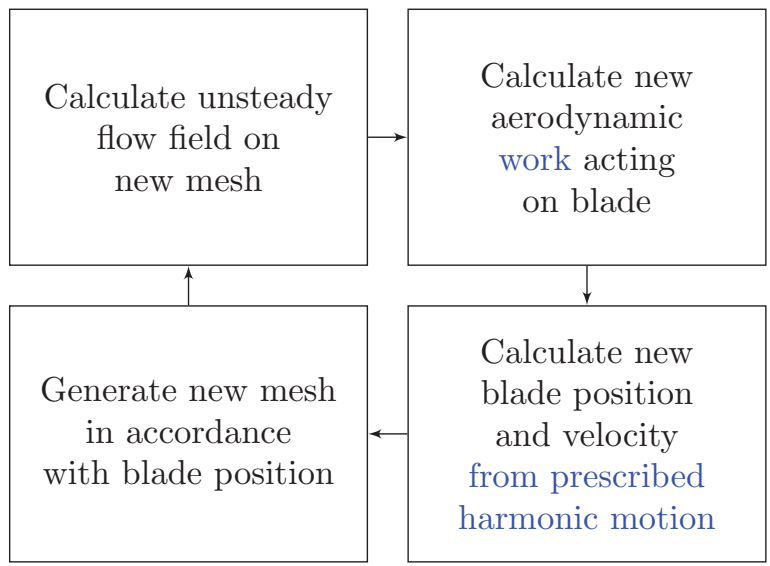

Figure 4: One-way coupling time marching scheme. spective annular position at the end of each oscillation cycle. In both plots of Fig. 5, the eleven-nodal diameter condition demonstrates the common occurrence whereby the blade may oscillate through a few cycles prior to reaching a converged state. As to be expected for a flow condition with axisymmetric conditions at the inlet plane, Fig. 5(b) shows great consistency about the annulus in a clean flow field. Analyzing blade-to-blade aerodynamic work data in the "unrolled" presentation of Fig. 5(b) proves to be particularly useful when studying a flow condition with inlet distortion.
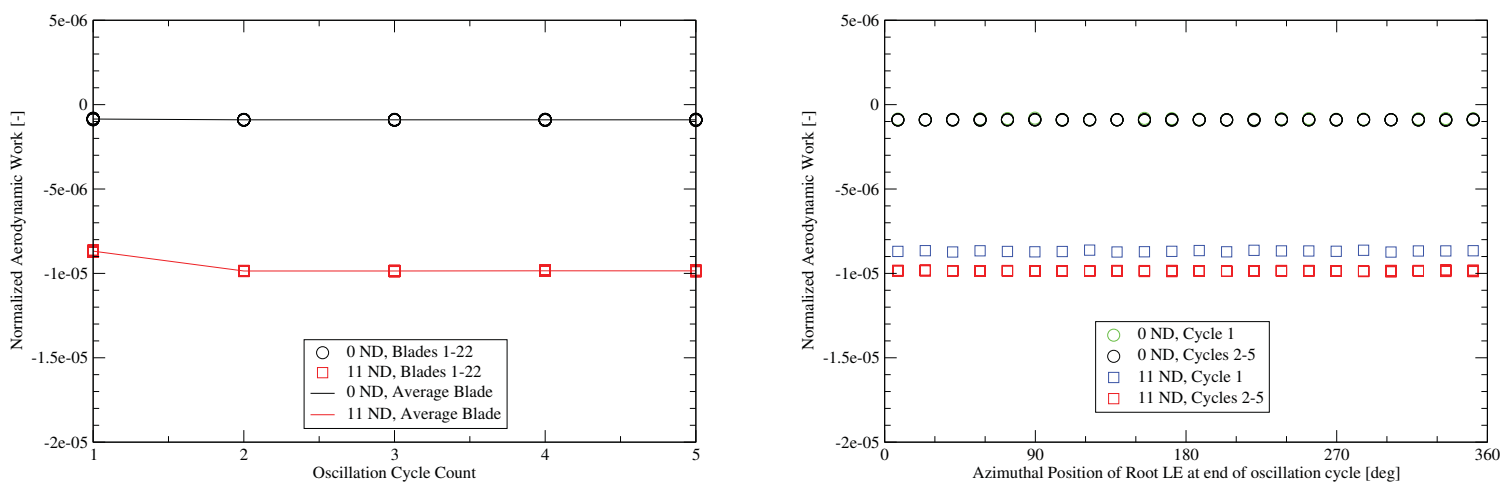

(a) Convergence of aerodynamic work for all blades ver- (b) Convergence of aerodynamic work for all blades versus oscillation cycle count. sus azimuthal position.

Figure 5: Time-convergence of aerodynamic work at part-speed, near op-line, clean inlet condition.

In the presence of circumferentially-varying distorted in-flow, the aerodynamic work done on/by the blade - and hence its damping - varies considerably as the blade traverses the flow field, see Fig. 6. Note that the ordinates are of identical scale among all plots in Figures 5 and 6 , providing perspective on the constancy of the aerodynamic work about the annulus for the clean inlet condition and the variation of the aerodynamic work about the annulus for the sinusoidally-distorted inlet condition. Observe further the close similarity between the converged "average" blade's aerodynamic work for both the clean inlet and the sinusoidally-distorted inlet. Recall that the the total pressure distribution of the distorted inlet applied here was specified to be a sinusoidal variation (of magnitude 4\%) about the clean inlet's radially-varying 
profile of total pressure. Close examination of the "average" blade's aerodynamic work quantities reveals the sinusoidally-distorted inlet condition to be marginally/negligibly less stable - but still stable - than the clean inlet condition near op-line.

In instances of inlet distortion wherein the aerodynamic work computations of the one-way coupling method vary from cycle to cycle and from blade to blade about the annulus - and all quantities indicate stability - it is conservative and reasonable to declare the system to be stable; in Fig. 6, the eleven-nodal pattern is clearly stable, and in the work of Bakhle ${ }^{26}$ et al. the system was clearly stable under these auspices. However, observe in Fig. 6(b) that a sector spanning approximately $150^{\circ}$ of the $360^{\circ}$ annulus exhibits unstable (positive) aerodynamic work for the zero-nodal diameter pattern. While the "average" blade indicates stability for zero nodal diameters, one cannot conservatively assert that the system is stable with the one-way coupling method consistently indicating instability over a defined sector of the annulus. Similar behavior, not shown here, is observed for for one-, two-, and three-nodal diameter forward traveling waves with inlet distortion near op-line.

Complemenary one-way coupling aeroelastic analyses are performed for the near-stall condition. Again, the "average" blade aerodynamic work computations (and hence aerodynamic dampings) are very similar between clean inlet and sinusoidally-distorted inlet conditions, with the sinusoidally-distorted inlet conditions usually yielding marginally/negligibly less stable dampings than the clean inlet for the part-speed, near-stall condition. For both the clean inlet and sinusodially- distorted inlet, the near-stall "average" blade dampings are less stable than the op-line "average blade" dampings. Notably, the 1 ND FTW (one nodal diameter forward traveing wave) pattern for the part-speed, near-stall condition yields small negative values of damping (i.e., flutter-unstable) for the "average" blade, whereby the sinsusoidally-distorted inlet is marginally/negligibly more unstable. As shown in Figure 7, for both clean inlet and distorted inlet for both one- and two-nodal diameter forward traveling waves, all blades alternate between stable (negative) aerodynamic work and unstable (positive) aerodynamic work as they circumnavigate the annulus. Each black line in Fig. 7(a) represents each individual blade as it traverses the annulus of the clean-inlet flow, while each red line plots the path of individual blades rotating through the distorted-inlet flow, for the one-nodal diameter forward traveling wave. Figure 7(b) shows similar behavior for the two-nodal diameter forward traveling wave.

Nodal diameter sweeps of "average" blade damping from zero-nodal diameter to three-nodal diameter forward-traveling wave for the part-speed condition - for both near-stall and near op-line, and for both clean inlet and distorted inlet - are plotted in Fig. 8. The eleven-nodal diameter pattern is strongly stable in all studies here; these data points are omitted from Fig. 8 for clarity of the data of nodal diameters zero through three.
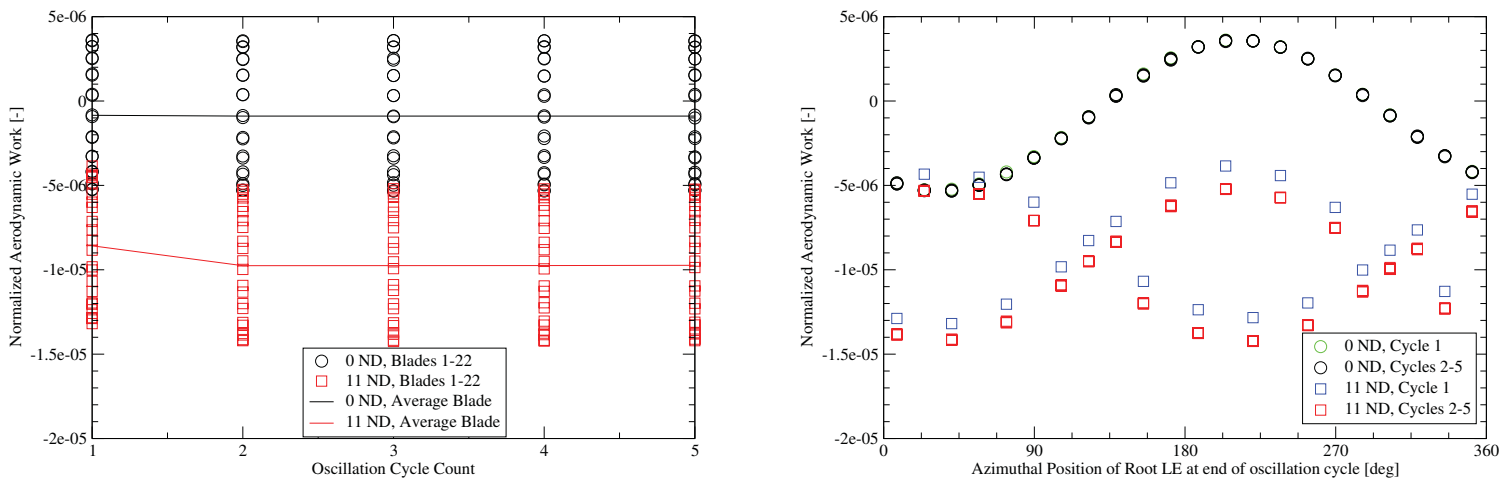

(a) Convergence of aerodynamic work for all blades ver- (b) Convergence of aerodynamic work for all blades versus oscillation cycle count. sus azimuthal position.

Figure 6: Time-convergence of aerodynamic work at part-speed, near op-line, sinusoidal distortion inlet condition. 


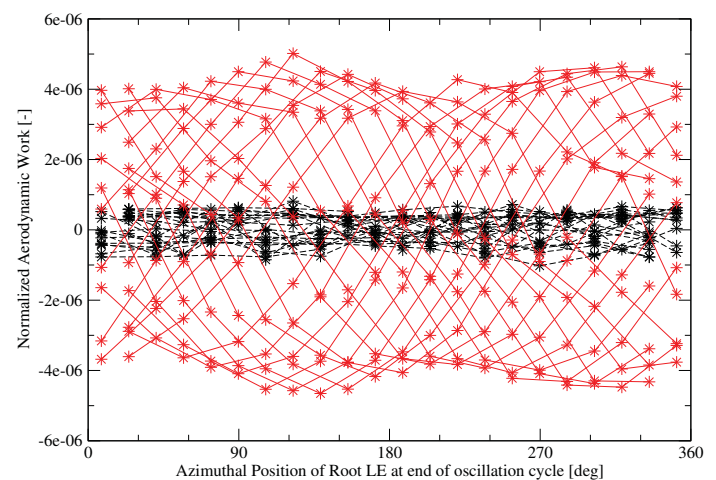

(a) 1 ND FTW

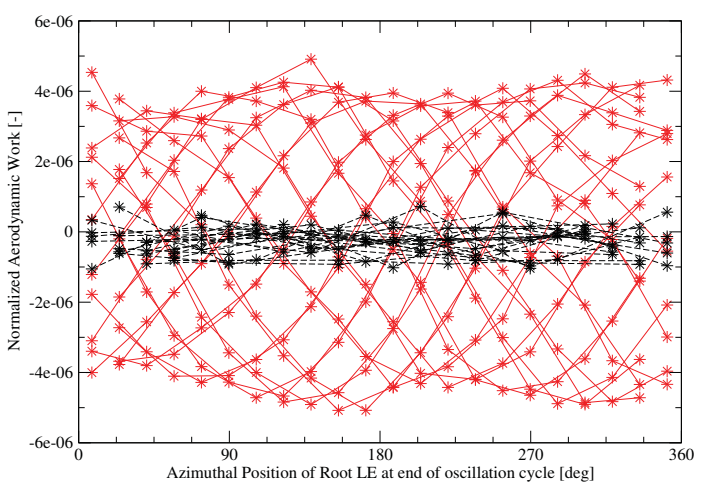

(b) 2 ND FTW

Figure 7: Aerodynamic work of all blades versus azimuthal position: Near stall, both clean (black) and distorted (red) inlets.

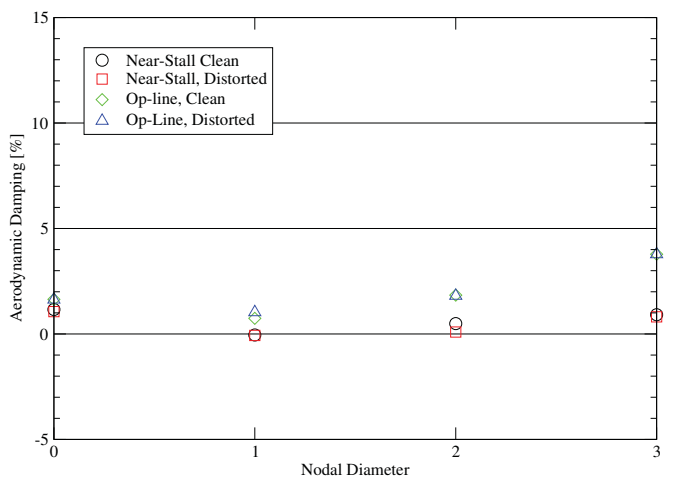

Figure 8: Aerodynamic damping versus nodal diameter using average-blade aerodynamic work from one-way coupled method.

\section{Two-way Coupled Method for Aeroelastic Analysis}

\section{A. Description and Implementation}

The two-way coupled method is implemented via a modal superposition framework similar to Gnesin and Rządkowski, ${ }^{10}$ Carstens $^{9}$ et al., and Bréard ${ }^{12}$ et al. In the two-way coupled method, the blade motion and the aerodynamic force acting on the blade each respond to, and act on, one another in sequential fashion in accordance with the equation of motion. Mode shapes are unit-mass normalized and hence modal stiffnesses equate to the squares of the respective frequencies. Modal forces are computed by taking the inner product of the blade surface pressure forces with the blade mode shape.

From the original equation of motion,

$$
[M]\{\ddot{X}\}+[C]\{\dot{X}\}+[K]\{X\}=\{F\}-\left\{f_{s}\right\}
$$

Pre-multiply by the mode shape $[\Phi]^{T}$, establish the generalized displacement coordinate $\eta$, and normalize to unit modal mass:

$$
\begin{aligned}
{[\Phi]^{T}[M][\Phi] } & =1 \\
\{X\} & =[\Phi]\{\eta\}
\end{aligned}
$$


Applying the conservative simplification of neglecting material and structural damping (i.e., assuming $C=0$ ). yields independent second order ordinary differential equations for each mode $i$ :

$$
\ddot{\eta}_{i}+\omega_{i}^{2} \eta_{i}=\left[\Phi_{i}\right]^{T}\left\{F_{i}-f_{s, i}\right\}
$$

Equation 6 represents the equation of motion for the blade in terms of its generalized displacement coordinate, $\eta$. This equation is time-integrated using a Newmark- $\beta$ method. ${ }^{30}$ This equation applies the modal forces, $F$, acting on the blades from the existing flow field (with the blades in their existing orientations) to compute the resulting deflections of the blades; these deflections are then applied to the blades to update the grid, and the aerodynamics and modal forces are recomputed and updated; this sequence is shown in Fig. 9.

For each computation and utilization of modal force in Eq. 6, a datum static modal force $f_{s}$ - representing the equilibrium loading on the undeformed hot blades - must be debited such that the blade vibrates about a mean equilibrium position corresponding to the undeformed hot blade. While for axisymmetric clean inlet flow the pressure forces acting on the blades are nominally constant as the blade circumnavigates the annulus, these pressure forces acting on the blade can vary significantly about the annulus in the presence of distorted in-flows, hence the computation and utilization of the static modal force demand careful consideration. This variation in unsteady pressures about the annulus is plotted in Fig. 10. Observe the considerable variation in blade loading - nearly $25 \%$ (mean-to-peak) near opline - as the blade traverses the annulus in the distorted flow (with an inlet total pressure variation of

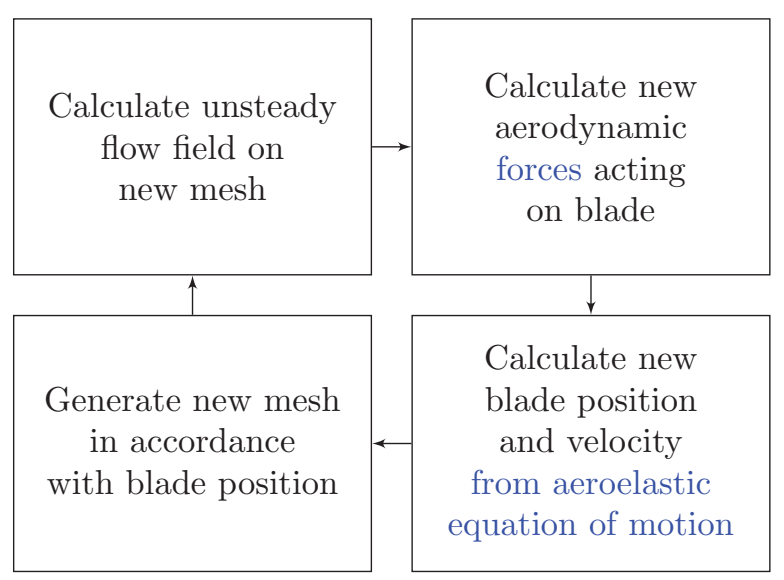

Figure 9: Two-way coupling time marching scheme. $4 \%$ mean-to-peak). As expected, the blade is more highly loaded near stall than near op-line. While the mean modal force for the distorted flow near op-line is almost equivalent to the nearly-constant modal force for the clean flow at the near op-line condition, there is greater difference between the mean modal force of distorted flow and the nearly-constant modal force of clean flow at the near stall condition. Also, while the variation in modal force within distorted flow near op-line is nearly sinusoidal, the variation in modal force within distorted flow near stall is more disorderly.

In the present implementation into TURBO, the two-way coupled method allows (or requires) userspecification of modal mass, stiffness or frequency of the mode(s), mechanical damping, initial blade displacements and velocities (or prescription of an initial nodal diameter pattern [or interblade phase angle] with displacement amplitude), and $\beta$ and $\gamma$ parameters for the Newmark- $\beta$ time integration. The user must also specify parameters of the "static modal force" for the simulation: retrieving modal force data on rigid blades over a period of one step, one blade pitch, or one revolution; and then sampling that static modal force data as discrete values, blade pitch averages, or full revolution averages. The fluid-structure coupling stencil applied in this study may be classified as conventional serial staggered; improved serial staggered ${ }^{31}$ and predictor-corrector stencils are in validation and shall be applied in future work.

\section{B. Validation}

While this two-way coupled method for aeroelastic analysis has been developed and implemented to facilitate greater insight into aeroelastic behavior of turbomachinery rotors in the presence of highly unsteady flows, it is instructive to first verify the performance of the algorithm in simpler, more fundamental aeroelastic environments. Given that the one-way coupled analysis procedure deflects the blades in a prescribed sinusoidal motion (irrespective of the aerodynamic modal forces acting on the blades), the two-way coupled analysis procedure should produce identical results when the computed modal forces are set to zero in the solution of Eq. 6. Figure 11 shows the requisite replication of the prescribed motion displacement at the blade tip, suction side, midchord (part-speed, clean inlet, near-stall shown) when using the two-way coupled motion procedure with modal forces neglected; this corroboration verfies that the Newmark- $\beta$ procedure adequately resolves the displacement-time evolution of the undamped elastic blade in a clean flow, near-stall environment. 


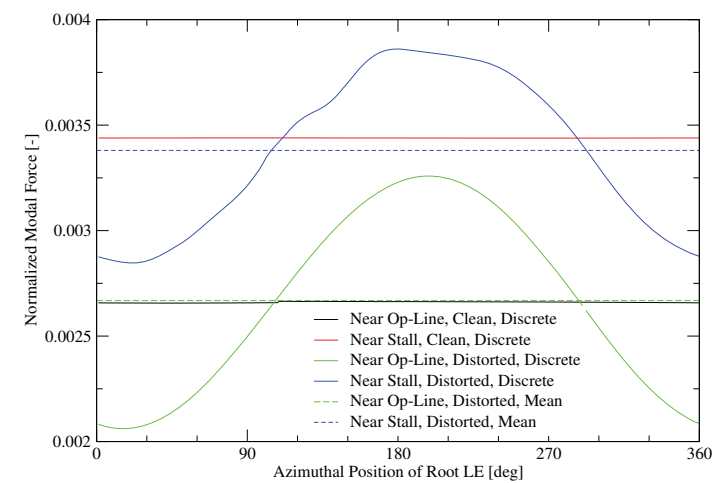

Figure 10: Static modal force: Modal force on rigid blades, due to unsteady pressure loading of clean and distorted flows. One blade shown.

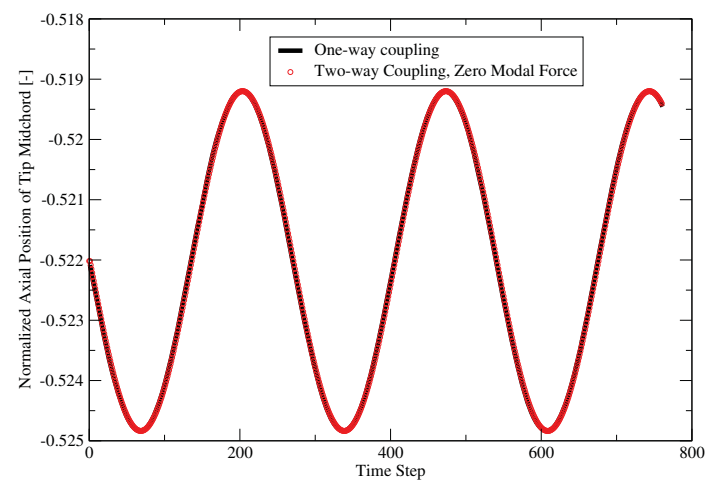

Figure 11: Blade axial motion (suction side tip, midchord) using both one-way prescribed motion procedure and two-way coupled motion procedure with modal forces zeroed.

Further validation of the method is evident from examination of its performance with its typical modal force coupling enforced. Figure 12 shows the blade's decreasing magnitude of oscillation in the 0 ND pattern near op-line in clean flow. The prescribed motion analysis conveys dampings of $1.64 \%$ for zero-nodal diameters (see Fig. 8), while logarithmic-decrement analysis of the tightly-coupled method's blade motion time histories yields dampings between $1.60 \%$ to $1.80 \%$. The positive aerodynamic damping conservatively indicates flutter stability.

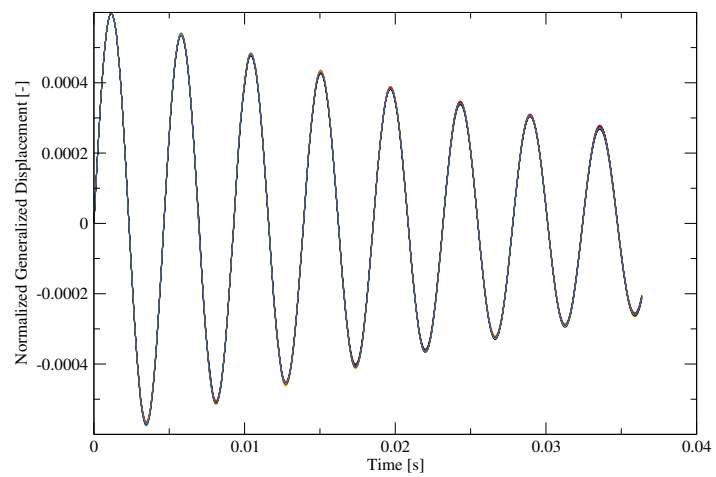

Figure 12: Two-way coupled simulation with decreasing magnitudes of blade oscillation for all blades, indicative of positive aerodynamic damping. Near op-line, clean-inlet flow, 0 ND initial condition. Static modal force: full revolution, sampled discretely.

\section{Application to Near-stall and Distorted Flows}

As discussed previously, the one-way coupling method clearly (and conservatively) shows flutter stability for this fan at part-speed, near op-line, clean inlet conditions. The flutter-stability question is less clear near stall for both clean and distorted inlet conditions, and there is evidence of periodic instability near op-line with distorted inlet conditions. The two-way coupled method shall be applied in further investigation of these fan operating conditions. For each of the following studies, the inlet conditions, exit conditions, fan speed, CFD time step, blade frequency, and blade mode shape are specified identically as for the one-way coupling 
runs. For highest fidelity - and with greatest computational expense - the static modal force application in these studies consists of compiling data from one revolution of fan operation with rigid blades, and then sampling this data discretely, matching the annular position of the deflecting blade (the current run) with the annular position of the static modal force database (a previous run with rigid blades but otherwise identical conditions).

\section{C.1. Near op-line, sinusoidal inlet distortion}

For a near op-line condition with a distorted inlet, the zero-nodal diameter pattern exhibits stability by inspection of the "average" blade, but unrolling the annulus reveals that a significant portion of the annulus lends itself to positive (unstable) aerodynamic work computations; see Fig. 6(b). The one-, two-, and threenodal diameter forward traveling wave patterns (not shown) have similar character in their flutter assessment via the one-way coupling method. Applying the two-way coupled analysis to the zero-nodal diameter pattern near op-line, it is readily evident that the blade is stable under these distorted inlet conditions; see Fig. 13. Comparison of Figures 12 and 13 shows little difference in damping for the zero-nodal diameter pattern, near op-line, between clean and distorted flows; these are nearly-identically stable conditions.

\section{C.2. Near stall, clean and distorted inlets}

Near stall, uncertainties regarding flutter stability arise in both clean and distorted flows with the one-way coupled method, due to the changes in sign of the aerodynamic work from blade to blade and from cycle to cycle; see Fig. 7(a). The one-way coupled method's "average" blade indicates negative aerodynamic damping (flutter instability) of $-0.068 \%$ and $-0.070 \%$ for clean and distorted flows, respectively, near stall (see Fig. 8). In Figures 14(a) and (b), displacements of all blades are plotted for both clean and distorted inlets for 1 ND FTW near stall. Figure 14(a) shows blade-to-blade inconsistency about the rotor with a small global trend of increasing blade displacements. Figure 14(b) shows great blade-to-blade inconsistency about the rotor and a greater global trend in the growth of blade displacements, indicating flutter instability.

The two-nodal diameter forward traveling wave pattern is assessed to be minimally stable near stall in both clean $(+0.49 \%$ damping $)$ and distorted $(+0.09 \%$ damping $)$ flows from the "average" blade computations using the one-way coupled method (see Fig. 8). Like the one-nodal diameter forward traveling wave, this two-nodal diameter pattern shows sign-changes about the annulus in its one-way coupled aerodynamic work computations; see Fig. 7(b). Figures 15(a) and (b) plot the displacements of all blades in both clean and distorted flows near stall upon application of the two-way coupled method. Like the 1 ND FTW case in clean flow, Fig. 15(a) shows blade-to- blade nonuniformity of displacements in clean flow; however, for the two-nodal diameter forward traveling wave pattern in clean flow, sporadic increases seem to damp again, as would be expected for the flutter-stable system predicted by the "average" blade using the one-way coupled method. For the 2 ND FTW in distorted flow, Fig. 15(b), the blades again exhibit nonuniformity of displacement about the annulus, but now the displacements seem to grow. While the one-way coupled method's "average" blade showed minimal stability in clean and distorted flows for 2 ND FTW near stall, the two-way coupled method shows stability near stall in clean flow but instability near stall in distorted flow.

\section{Conclusions and Future Work}

An initial comparison between one-way and two-way coupled flutter analysis methods implemented in TURBO has been performed. An existing fan, for which TURBO's one-way coupled flutter analysis method has been experimentally corroborated, was used as the computational test article. The fan's structural dynamics characterstics (mode shape and natural frequency) were altered, and the fan was run at part-speed conditions with intention of simulating stable and unstable flutter environments. The fan was provided with both axisymmetric inlet total pressure as well as a sinusoidal variation of total pressure about the annulus, such that the integrated, mean flow parameters for the distorted flow were equivalent to the clean flow, and thus the circumferential uniformity (or nonuniformity) of the inlet flow would be the only difference between the two flow conditions.

As had been demonstrated previously, TURBO's one-way coupling method provided consistent work-percycle computations for all blades across the annulus in clean flow, while distorted flow caused great variation in aerodynamic work computations from blade to blade and from cycle to cycle. The "average" blade can 


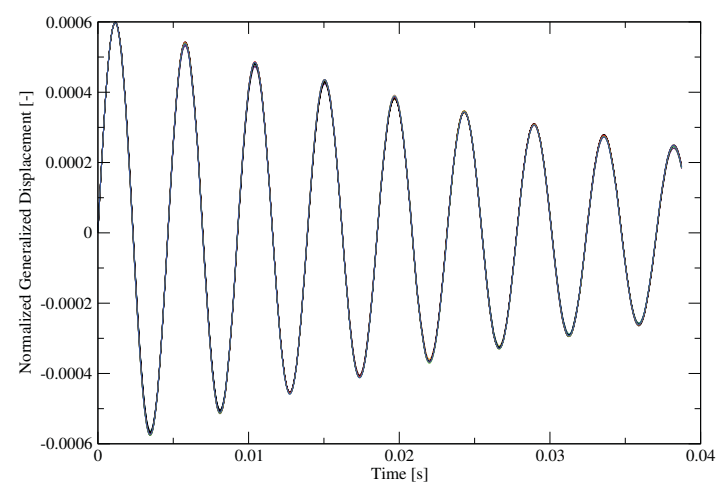

Figure 13: Decreasing magnitudes of oscillation of all blades, indicating flutter stability. Near op-line, distorted inlet, 0 ND initial condition. Static modal force: full revolution sampled discretely.

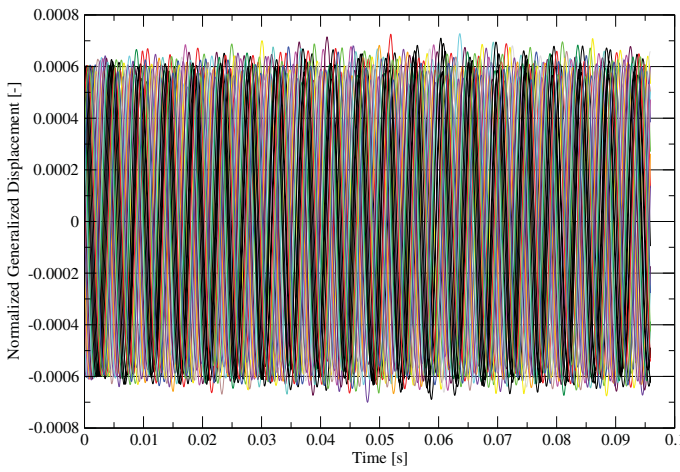

(a) Near stall, clean inlet, 1 ND FTW, all blades.

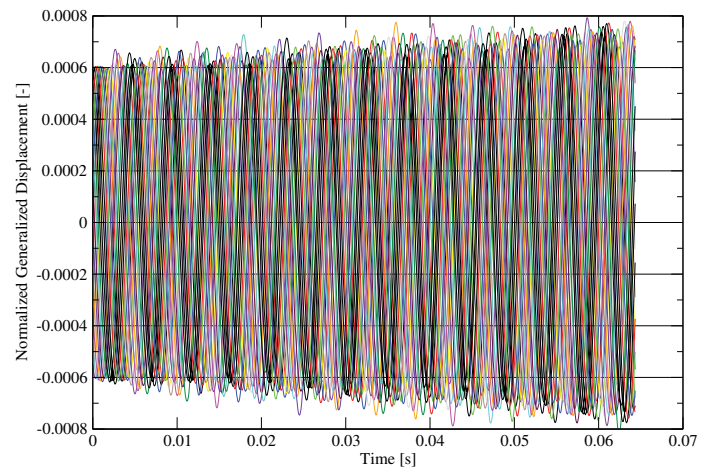

(b) Near stall, distorted inlet, 1 ND FTW, all blades

Figure 14: Comparing blade displacements at near-stall, 1 ND FTW, for both clean and distorted inlets. Static modal force: full revolution sampled discretely.

be conservatively applied to assess flutter stability in clean flow, provided that all blades' aerodynamic work computations indicate stability. Multiple cases were observed wherein the "average" blade was stable, but the full annulus of blades would oscillate between stability and instability from blade to blade and from cycle to cycle in an orderly repeating sequence.

The two-way coupling method corroborated the one-way coupling method in assessing flutter stability in clean flow near op-line. The two-way coupling method was then used to investigate the flutter stability of clean flow near stall as well as distorted flow at both near op-line and near stall conditions. In most cases, the two-way coupling corroborated the adequacy of the one-way coupling method's "average" blade assessment of flutter stability. In general, the distorted flow was slightly less stable (or slightly more unstable) than its clean flow counterpart. However, for 1 ND FTW and 2 ND FTW at near stall conditions, the "average" blade's (one-way coupling) aerodynamic work oscillated between small values positive and negative in clean flow and among large values positive and negative in distorted flow. The two-way coupled method showed blade-to-blade variation at these conditions, but 1 ND FTW seemed to be minorly unstable in clean flow and more unstable in distorted flow. Most notably, the two-way coupled analysis of 2 ND FTW showed to be stable in clean flow but unstable in distorted flow.

While instabilities seem to have been demonstrated, their magnitude was quite small, and these must be investigated further. Refined gridding, refined time-stepping, and longer simulation times would be 


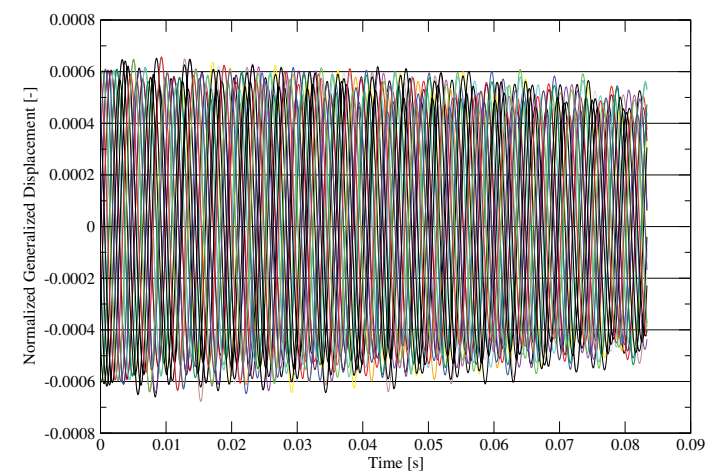

(a) Near stall, clean inlet, 2 ND FTW, all blades.

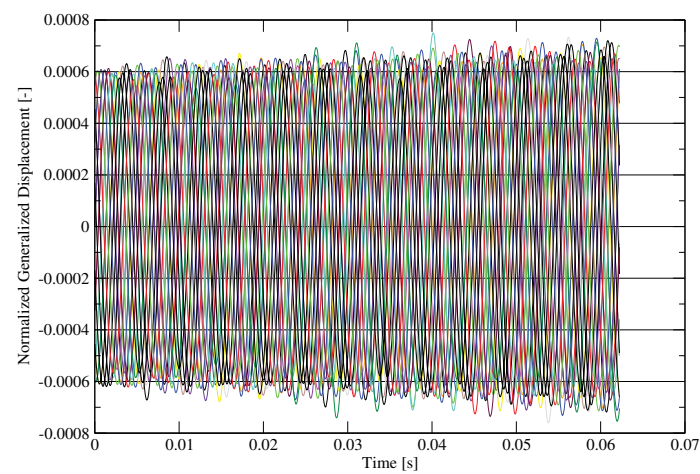

(b) Near stall, distorted inlet, 2 ND FTW, all blades

Figure 15: Comparing blade displacements at near-stall, 2 ND FTW, for both clean and distorted inlets. Static modal force: full revolution sampled discretely.

conducive to more in-depth study of the fluid mechanics' role in these potential instabilities. The timestep for these simulations, though constant between one-way and two-way coupling simulations, was rather coarse. Different fluid-structure coupling stencils exist which could prove critical when operating so closely to neutral stability. All two-way coupled flutter simulations discussed herein used a full revolution of "static modal force" data and sampled it discretely because it was thought to offer the highest fidelity (at the highest computational cost) of the fluid-structure interaction; the "static modal force" data is generated by running a rigid-blade simulation with identical parameters to the subsequent elastic-blade simulation of primary interest. NASA's work in researching developing BLI propulsion systems continues, and in time there will be experimental aeromechanics data of BLI devices to complement and validate computational method development.

\section{Acknowledgments}

The author appreciates the many valuable discussions with Dr. Milind A. Bakhle (NASA Glenn Research Center) through the course of this work. The author is grateful for the support of this work provided by the NASA Fixed Wing Project: Dr. Rubén Del Rosario, Manager; Dr. Michael D. Hathaway, Technical Lead; and Mr. James F. Walker, Deputy Project Manager for Glenn Research Center.

\section{References}

${ }^{1}$ Smith, L. H., "Wake Ingestion Propulsion Benefit," AIAA Journal of Propulsion and Power, Vol. 9, No. 1, Jan-Feb 1993, pp. 74-82.

${ }^{2}$ Daggett, D., Kawai, R., and Friedman, D., "Blended Wing Body System Studies: Boundary Layer Ingerstion Inlets with Active Flow Control," Tech. Rep. NASA/CR-2003-212670, Dec. 2003.

${ }^{3}$ Kawai, R., Friedman, D., and Serrano, L., "Blended Wing Body (BWB) Boundary Layer Ingestion (BLI) Inlet Configuration and System Studies," Tech. Rep. NASA/CR-2006-214534, Dec. 2006.

${ }^{4}$ Plas, A. P., Sargeant, M. A., Madami, V., Crichton, D., Greitzer, E. M., Hynes, T. P., and Hall, C. A., "Performance of a Boundary Layer Ingesting (BLI) Propulsion System," 45th AIAA Aerospace Sciences Meeting and Exhibit, No. 2007-AIAA0450, Jan. 2007.

${ }^{5}$ Nickol, C. L., "Silent Aircraft Initiative Concept Risk Assessment," Tech. Rep. NASA/TM-2008-215112, Feb. 2008.

${ }^{6}$ Nickol, C. L. and McCullers, L. A., "Hybrid Wing Body Configuration System Studies," 47th AIAA Aerospace Sciences Meeting Including The New Horizons Forum and Aerospace Exposition, No. 2009-AIAA-0931, Jan. 2009.

${ }^{7}$ Tillman, T. G., Hardin, L. W., Moffitt, B. A., Sharma, O. P., Lord, W. K., Berton, J., and Arend, D., "System-Level Benefits of Boundary Layer Ingesting Propulsion," AIAA 49th Aerospace Sciences Meeting, Jan. 2011, Invited presentation.

${ }^{8}$ Herrick, G. P., "Effects of Inlet Distortion on Aeromechanical Stability of a Forward-Swept High-Speed Fan," 46th AIAA/ASME/SAE/ASEE Joint Propulsion Conference \& Exhibit, No. 2010-AIAA-6711, July 2010.

${ }^{9}$ Carstens, V., Kemme, R., and Schmitt, S., "Coupled simulation of flow-structure interaction in turbomachinery," Aerospace Science and Technology, Vol. 7, 2003, pp. 298-306. 
${ }^{10}$ Gnesin, V. and Rządkowski, R., "A Coupled Fluid-Structure Analysis for 3-D Inviscid Flutter of IV Standard Configuration," Journal of Sound and Vibration, Vol. 251, No. 2, 2002, pp. 315-327.

${ }^{11}$ Marshall, J. G. and Imregun, M., "An Analysis of the Aeroelastic Behaviour of a Typical Fan Blade with Emphasis on Flutter Mechanisms," International Gas Turbine and Aeroengine Congress 85 Exposition, No. ASME 96-GT-78.

${ }^{12}$ Bréard, C., Vahdati, M., Sayma, A. I., and Imregun, M., "An Integrated Time-Domain Aeroelasticity Model for the Prediction of Fan Forced Response due to Inlet Distortion," Journal of Engineering for Gas Turbines and Power, Vol. 124, No. 1, 2002, pp. 196-208.

${ }^{13}$ Zhu, J. and Shih, T. H., "CMOTT Turbulence Model for NPARC," Tech. Rep. NASA/CR-1997-204143, Aug. 1997.

${ }^{14}$ Chen, J.-P. and Whitfield, D. L., "Navier-Stokes Calcuations for the Unsteady Flowfield of Turbomachinery," Tech. Rep. 1990-AIAA-0676, Jan. 1990.

${ }^{15}$ Chen, J.-P. and Briley, W. R., "A Parallel Flow Solver for Unsteady Multiple Blade Row Turbomachinery Simulations," Proceedings of ASME TURBO EXPO 2001, No. GT-2001-0348, June 2001.

${ }^{16}$ Srivastava, R., Bakhle, M. A., and Keith, Jr., T. G., "Numerical Simulation of Aerodynamic Damping for Flutter Analysis of Turbomachinery Blade Rows," AIAA Journal of Propulsion and Power, Vol. 19, No. 2, March 2003.

${ }^{17}$ Srivastava, R., Bakhle, M. A., Keith, Jr., T. G., and Stefko, G. L., "Flutter Analysis of a Transonic Fan," Proceedings of ASME TURBO EXPO 2002, No. GT2002-30319, June 2002.

${ }^{18}$ Bakhle, M. A., Liu, J. S., Panovsky, J., Keith, Jr., T. G., and Mehmed, O., "Calculation and Correlation of the Unsteady Flowfield in a High Pressure Turbine," Proceedings of ASME TURBO EXPO 2002, No. GT2002-30322, June 2002.

${ }^{19}$ Panovsky, J., James, D. K., and Hassan, K. K., "An Assessment of Fan Flutter Using TURBO," 5th National Turbine Engine High Cycle Fatigue Conference, Universal Technology Corporation, Dayton, Ohio, April 2000.

${ }^{20}$ Sanders, A. J., "Stall Flutter Assessment of an Advanced Design Transonic Fan Using TURBO-AE," 6th National Turbine Engine High Cycle Fatigue Conference, Universal Technology Corporation, Dayton, Ohio, April 2001.

${ }^{21}$ Panovsky, J., Liu, J. S., and Bakhle, M. A., "Comparisons of Experimental and Computational Forced Response in a High Pressure Turbine," 7th National Turbine Engine High Cycle Fatigue Conference, Universal Technology Corporation, Dayton, Ohio, April 2002.

${ }^{22}$ Kielb, R. E., Barter, J. W., Thomas, J. P., and Hall, K. C., "Blade Excitation by Aerodynamic Instabilities," Proceedings of ASME TURBO EXPO 2003, No. GT2003-38634, June 2003.

${ }^{23}$ Sanders, A. J., Hassan, K. K., and Rabe, D. C., "Experimental and Numerical Study of Stall Flutter in a Transonic Low-Aspect Ratio Fan Blisk," ASME Journal of Turbomachinery, Vol. 126, No. 1, 2004, pp. 166-174.

${ }^{24}$ Sanders, A. J., "Nonsynchronous Vibration (NSV) due to a Flow-Induced Aerodynamic Instability in a Composite Fan Stator," ASME Journal of Turbomachinery, Vol. 127, No. 2, 2005, pp. 412-421.

${ }^{25}$ Bakhle, M. A., Reddy, T. S. R., and Stefko, G. L., "Comparisons of Flutter Analyses for an Experimental Fan," Tech. Rep. NASA/TM-2010-216221, May 2010.

${ }^{26}$ Bakhle, M. A., Reddy, T. S. R., Herrick, G. P., Shabbir, A., and Florea, R., "Aeromechanics Analysis of a Boundary Layer Ingesting Fan," 48th AIAA/ASME/SAE/ASEE Joint Propulsion Conference \& Exhibit, No. AIAA-2012-3995, 2012.

${ }^{27}$ Berrier, B. L., Carter, M. B., and Allan, B. G., "High Reynolds Number Investigation of a Flush-Mounted, S-Duct Inlet With Large Amounts of Boundary Layer Ingestion," Tech. Rep. NASA/TP-2005-213766, Sept. 2005.

${ }^{28}$ Carta, F. O., "Coupled Blade-Disk Shroud Flutter Instabilities in Turbojet Engine Rotors," ASME Journal of Engineering for Power, July 1967, pp. 419-426.

${ }^{29}$ Vasanthakumar, P., "Computation of Aerodynamic Damping for Flutter Analysis of a Transonic Fan," Proceedings of ASME TURBO EXPO 2011, No. GT2011-45697, June 2011.

${ }^{30}$ Newmark, N. M., "A method of computation for structural dynamics," ASCE Journal of Engineering Mechanics, Vol. 8, 1959, pp. 67-94.

${ }^{31}$ Farhat, C. and Lesoinne, M., "Two efficient staggered algorithms for the serial and parallel solution of three-dimensional nonlinear transient aeroelastic problems," Computational Methods in Applied Mechanics and Engineering, Vol. 182, 2000, pp. $499-515$. 